\title{
ANORMALIDAD FLORAL EN PHYTOLACCA ICOSANDRA L. (PHYTOLACCACEAE) EN EL PEDREGAL DE SAN ÁNGEL, MÉXICO, D.F.
}

\author{
Ramiro Cruz Durán \\ Y \\ Othón Alcántara Ayala \\ Herbario FCME \\ Departamento de Biología \\ Facultad de Ciencias \\ Universidad Nacional Autónoma de México \\ Apartado postal 70-399 \\ 04510 México D.F. \\ e-mail: cdr@minervaux2.fciencias.unam.mx
}

\section{RESUMEN}

Se describen las anormalidades florales presentes en varios individuos de Phytolacca icosandra L. del Pedregal de San Ángel, en áreas cercanas a los edificios de la Facultad de Ciencias de la Universidad Nacional Autónoma de México, las cuales anteriormente albergaban un matorral xerófilo de Senecio praecox L. ("palo loco"), pero que ahora se encuentran en proceso de urbanización.

Las anormalidades consisten principalmente en el alargamiento de la base del ovario a manera de un ginóforo, así como en la transformación de los sépalos, anteras y carpelos en estructuras semejantes a hojas. En casos más extremos, a partir del interior del ovario surge una nueva rama.

\section{ABSTRACT}

Cases of flower abnormalities are described in several individuals of Phytolacca icosandra L. that inhabit the Pedregal de San Angel, near the buildings of the Faculty of Sciences of the Universidad Nacional Autónoma de México, which previously harbored a scrub of Senecio praecox L. ("palo loco") and now is in process of urbanization.

The abnormalities consist mainly in the enlargement of the base of the ovary, which takes the appearance of a gynophore, as well as in the transformation of sepals, anthers and carpels into structures similar to leaves. In extreme cases a new branch emerges from the interior of the ovary.

\section{INTRODUCCIÓN}

Los casos registrados de anormalidades florales, al contrario de lo que se pudiera pensar, no son escasos, ya que todos los órganos o estructuras de la flor tienen la capacidad de transformarse en otros órganos, como los que presenta una flor normal e incluso en un órgano no floral. 
Este tipo de fenómenos se han registrado al menos desde 1830 (Seringe, 1830; Mohl, 1837); habiéndose publicado listas de plantas que en ocasiones presentan algún tipo de anormalidad en la flor (Meyer, 1966). Los trabajos sobre anormalidades de flores son particularmente útiles para entender los procesos de diferenciación vegetal y en los estudios de tipo evolutivo (Sussex y Kerk, 1990).

Existen diversas clases de anormalidades florales, algunas de las más frecuentes son la filodia, la sepalodia, la petalodia, la estaminodia y la carpelodia (Meyer, 1966); cada uno de estos términos hace referencia al tipo de estructura en la que se llegan a convertir los órganos florales.

Pueden ser varios los agentes causantes de las anormalidades mencionadas; en ocasiones las variaciones climáticas extremas modifican el desarrollo de la flor. Otros factores del ambiente, tales como la disponibilidad de nutrientes o la humedad del suelo, pueden afectar también la diferenciación. En algunas ocasiones, incluso fenómenos de parasitismo pueden ser la causa de la anormalidad. En muchos casos experimentales el tratamiento químico basado en auxinas, giberelinas y citoquininas principalmente, ha sido utilizado para modificar el desarrollo vegetal, incluyendo el de la flor (Diomaiuto, 1988; Zeevaart, 1978). Las causas de las anormalidades florales son también de tipo genético (Sussex y Kerk, 1990). Así, se han registrado mutantes en genes homeóticos (desarrollo de órganos en una posición no usual) en flores de Antirrhinum y Arabidopsis (Coen y Meyerowitz, 1991).

\section{LOCALIDAD DE LOS EJEMPLARES}

Los casos de anormalidad floral estudiados se presentan en varios individuos de Phytolacca icosandra L. que habitan en áreas muy pequeñas y de díficil delimitación, que generalmente se encuentran dispersas entre los diferentes edificios que limitan con la parte este de la Facultad de Ciencias, en la Ciudad Universitaria (UNAM). La Ciudad Universitaria se ubica en el Pedregal de San Ángel, corriente de lava basáltica en la que existen numerosos macro y microambientes, mismos que han resultado favorables para el establecimiento de una gran cantidad de especies vegetales y donde se registran seis asociaciones vegetales, entre ellas el Senecionetum praecocis (matorral de Senecio praecox L.), dentro de los matorrales xerófilos. Originalmente esta comunidad cubría una superficie aproximada de $40.45 \mathrm{~km}^{2}$ y en la actualidad, debido al incesante avance del urbanismo, se restringe a $2.9 \mathrm{~km}^{2}$ (Álvarez et al., 1989).

La población mejor representada de Phytolacca icosandra L. en el área se encuentra hacia los 19¹9'26" de latitud N y 99¹0'42" de longitud W, a 2240 m de altitud, ocupando una superficie aproximada de $200 \mathrm{~m}^{2}$ y está formada por 22 individuos de los cuales, cuatro presentan anormalidades florales. El suelo donde se desarrolla esta población es superficial y se halla por encima de una capa formada por roca basáltica de origen volcánico; está constituido por los productos de descomposición eólica de la lava y por los depósitos orgánicos de las plantas que habitan el área. 
Cruz y Alcántara: Anormalidad Floral en Phytolacca icosandra en el Pedregal de San Ángel

\section{DESCRIPCIÓN DE LA FLOR NORMAL DE LA ESPECIE}

Considerando que las anormalidades observadas afectan exclusivamente a las flores, se describen a continuación las características de las estructuras florales normales con base en la consulta de ejemplares de herbario.

Phytolacca icosandra L. se presenta en el área de estudio como una hierba sufruticosa, de hasta $1.6 \mathrm{~m}$ de altura; inflorescencias en racimos erectos, opositifoliares, de 9 a $24 \mathrm{~cm}$ de largo por 0.6 a $1.2 \mathrm{~cm}$ de ancho, con aprox. 80 flores y frutos por racimo, pedúnculos de 2.5 a $4 \mathrm{~cm}$ de largo, pedicelos de 0.2 a $0.3 \mathrm{~cm}$ de largo, brácteas de 0.2 a $0.4 \mathrm{~cm}$ de largo, márgenes rojizos, lanceolados, bracteolas rojizas subuladas, de menos de $1 \mathrm{~mm}$, pedicelos ligeramente glabrescentes, flores alternas; tépalos persistentes en la antesis, verdes con el margen rojizo, después de la antesis de color carmín o rojo púrpura, acrescentes, obovados, de 0.2 a 0.3 de largo por 0.1 a $0.2 \mathrm{~cm}$ de ancho, ápice agudo a redondeado, base angosta; estambres 12 a 20, en dos series, filamentos blancos, de $0.2 \mathrm{~cm}$ de largo, anteras amarillas, de $1 \mathrm{~mm}$ de largo, oblongas, con apertura longitudinal; ovario con 8 a 10 carpelos connados en la antesis, placentación axilar, estilos unidos en la base y libres en el ápice, persistentes (Fig. 1).

La especie se distribuye desde el norte de México a través de Centroamérica, hasta Venezuela y Ecuador, así como en las Antillas. En nuestro país se encuentra de preferencia en el bosque tropical caducifolio, bosque de Pinus y Abies, bosque de Quercus, comúnmente en la vegetación secundaria (Martínez-García, 1984).

Ejemplares utilizados para elaborar la descripción. Distrito Federal: El Ajusco, velveder, E del Ajusco, C. Solórzano T. s/n (FCME); Pedregal de San Ángel, C. Cabello $s / n$ (FCME); Sierra del Chichinautzin, $5 \mathrm{~km}$ al $\mathrm{S}$ de Álvaro Obregón, carretera al Ajusco, L. Silvestry U. s/n (FCME); Pedregal de San Ángel, O. Alcántara A. s/n (FCME); Facultad de Ciencias, UNAM, entre los edificios A y B de Biología, R. Cruz D. 4573 (FCME); Parque Lira, H. Vibrans 4680 (MEXU); Calle Fernando Villalpando, H. Vibrans 4397 (MEXU); 1 km al N de La Venta, Desierto de Los Leones, R. Fernández N. 409 (MEXU); Santa Cecilia, Delegación Xochimilco, A. Ventura A. 2793 (MEXU); Ejidos de Padierna, M. A. Panti M. 546 (MEXU); San Antonio Tecómitl, Milpa Alta, R. M. Yedera V. 7 (MEXU); Ticomán, Cuautepec, F. Gallegos H. 94 (MEXU); Pedregal, D.F., E. Lyonnet 321 (MEXU); Jardín Botánico, UNAM, A. García M. 4773 (MEXU).

\section{ANORMALIDADES FLORALES OBSERVADAS}

Son varias las anormalidades que se registraron en la flor de Phytolacca icosandra L.; en uno de los casos se observa un alargamiento del ovario, que es más acentuado en su parte basal, adoptando esta última la forma similar a un ginóforo, el cual varía en tamaño de 0.4 a $1.7 \mathrm{~cm}$ de largo (Fig. 2A). Los tépalos presentan también un aumento en su tamaño, tomando el aspecto de hojas de 1 a $1.5 \mathrm{~cm}$ de largo. Los estambres, tanto los filamentos como las anteras, se tornan verdes. En una variante de este caso, el ovario alargado se abre y cada uno de los carpelos se transforma en una hoja (Fig. 2B). 
Acta Botanica Mexicana (2000), 53: 27-33

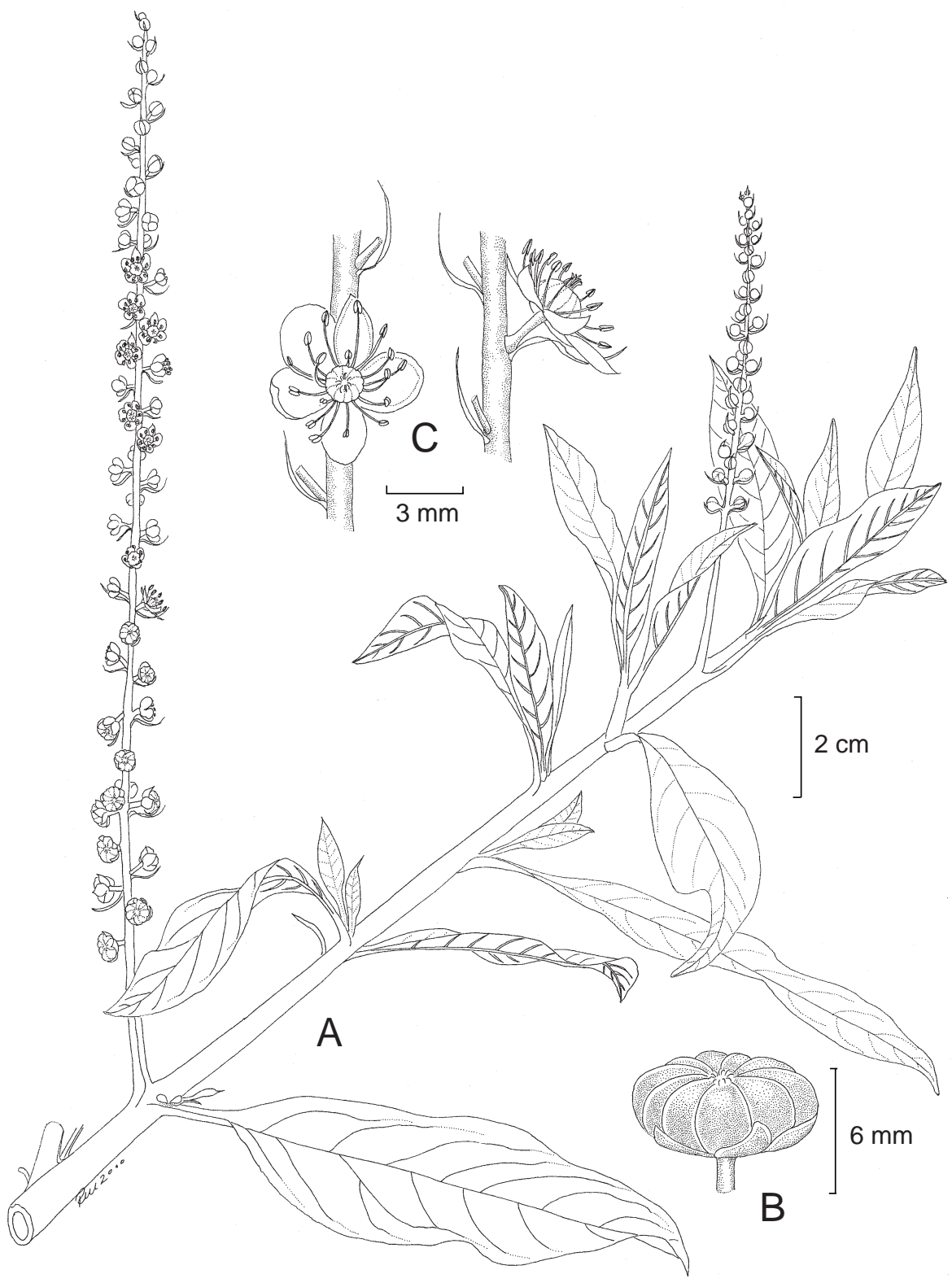

Fig. 1. Planta normal de Phytolacca icosandra L. A. Rama con inflorescencias; B. Fruto; C. Flor. (Basado en R. Cruz D. 4573). 
Cruz y Alcántara: Anormalidad Floral en Phytolacca icosandra en el Pedregal de San Ángel
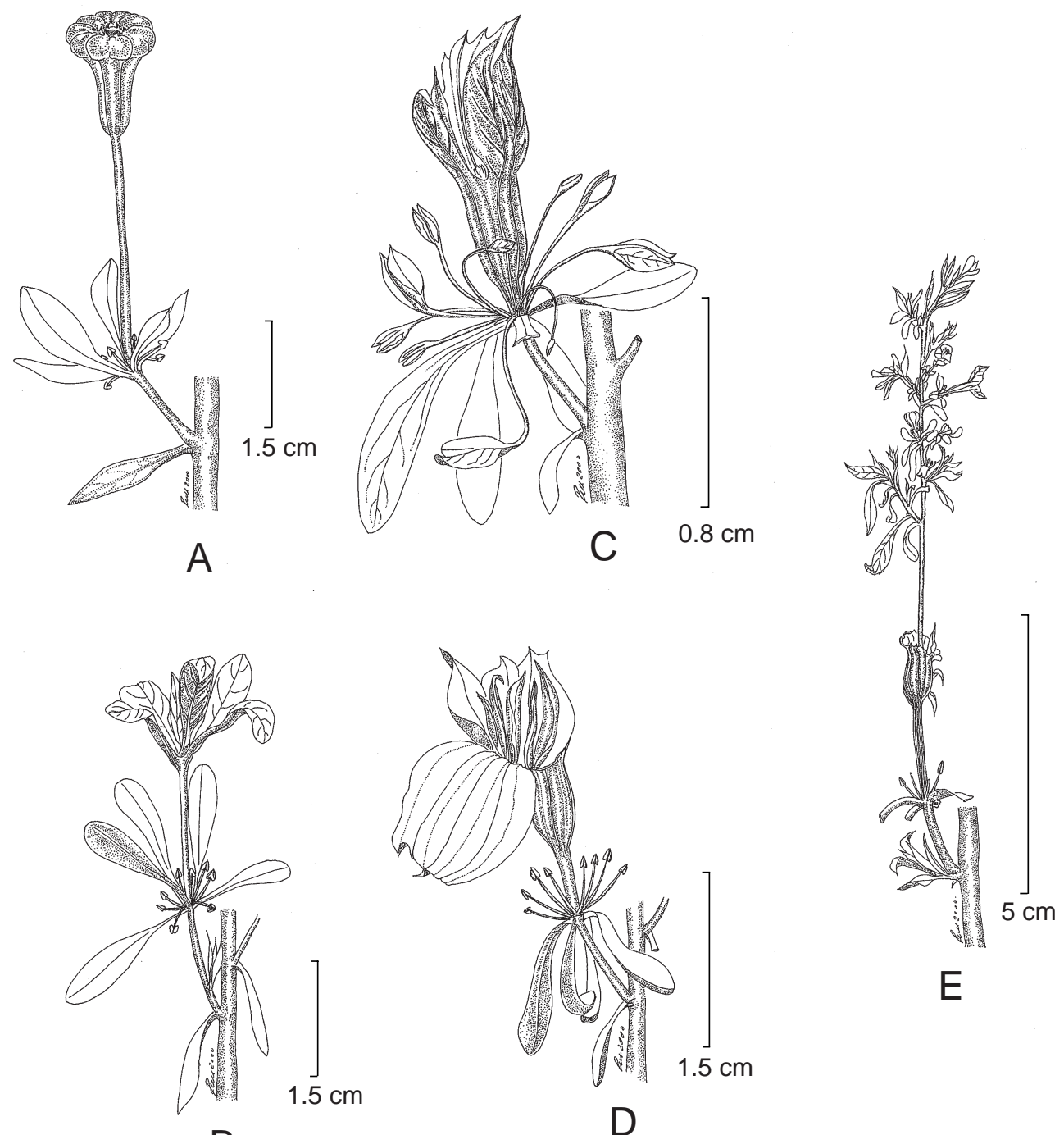

B

Fig. 2. Flores anormales de Phytolacca icosandra L. A. Flor con la base del ovario alargada a manera de un ginóforo; B. Flor también con la base del ovario alargada, pero con los carpelos y tépalos transformados en estructuras parecidas a hojas; C. Flor anormal con los estambres transformados en hojas pequeñas; D. Ovario transformado en una especie de "copa" con estructuras internas foliosas; E. Ovario anormal, de cuyo interior surge una nueva rama la cual ha producido nuevas yemas y flores anormales. (A, basado en R. Cruz D. 4574; B, C, D y E, basados en R. Cruz D. s/n). 
En otras flores, además del alargamiento de los tépalos y del ovario, que no es tan acentuado en su base como en el caso anterior, los filamentos de los estambres crecen en longitud hasta $0.5 \mathrm{~cm}$, las anteras se abren por las suturas en cada teca, y éstas se aplanan y adquieren el aspecto de una hoja pequeña, en tanto que el filamento se torna verde y se asemeja a un pecíolo (Fig. $2 \mathrm{C}$ ). También se da el caso en el que los componentes internos del ovario (funículo, óvulos) igualmente se alargan y se transforman en estructuras foliosas, mientras que cada carpelo se abre desde la parte apical a lo largo de las suturas, conformando una especie de copa plegada que rodea a estas porciones internas (Fig. 2D).

En los casos más extremos, se observa que a partir del interior del ovario surge una rama, la cual presenta yemas, flores anormales, así como nuevas ramas. En estos individuos los estambres no sufren grandes modificaciones, sólo se tornan verdes (Fig. 2E).

Es importante resaltar el gran parecido que existe entre los ejemplares que presentan algunas de estas anormalidades con las plantas descritas como Nowickea, género propuesto como nuevo para la familia Phytolaccaceae por Martínez-García y McDonald (1989). Estas semejanzas son notables sobre todo si se comparan los ginóforos, los ovarios y los sépalos alargados de los ejemplares ilustrados en el mencionado trabajo.

Ejemplares con anormalidades examinados. Distrito Federal: Facultad de Ciencias, UNAM, entre el invernadero y la biblioteca "Ricardo Monges López", 26.III.2000, R. Cruz D. s/n (FCME); frente al postgrado de Química, UNAM, 21.I.2000, R. Cruz D. 4574 (FCME); Facultad de Ciencias, UNAM, frente al invernadero, 10.XI.1997, R. Cruz D. s/n (FCME); Facultad de Ciencias, UNAM, a un costado del invernadero, 27.III.1997, R. Cruz D. s/n (FCME); Facultad de Ciencias, UNAM, entrada del estacionamiento de estudiantes, 00.III.2000, O. Alcántara A. s/n (FCME).

\section{AGRADECIMIENTOS}

Se agradece al M. en C. Jaime Jiménez Ramírez y a la M. en C. Aurora Zlotnik la revisión crítica del manuscrito y sus valiosas sugerencias, así como a los curadores de los herbarios FCME y MEXU por las facilidades en la consulta de los ejemplares. Dos revisores anónimos hicieron importantes recomendaciones.

\section{LITERATURA CITADA}

Álvarez, J., J. Carabias, J. Meave, P. Moreno-C., D. Nava, F. Rodríguez, C. Tovar y A. Valiente. 1989. Proyecto para la creación de una reserva en el pedregal de San Ángel. Laboratorio de Ecología. Facultad de Ciencias, Universidad Nacional Autónoma de México. México, D.F. 54 pp.

Coen, E. S. y E. M. Meyerowitz. 1991. The war of the whorls: genetic interactions controlling flower development. Nature 353: 31-37.

Diomaiuto, J. 1988. Periodic flowering or continual flowering as a function of temperature in a perennial species: The ravenelle wall flower (Cheiranthus cheiri). Phytomorphology 38(2,3): 163-171.

Martínez-García, J. 1984. Phytolaccaceae. Flora de Veracruz. Instituto Nacional de Investigaciones sobre Recursos Bióticos. Xalapa, Ver. 41 pp.

Martínez-García, J. y J. A. McDonald. 1989. Nowickea (Phytolacacceae), a new genus with two new species from Mexico. Brittonia 41(4): 399-403. 
Cruz y Alcántara: Anormalidad Floral en Phytolacca icosandra en el Pedregal de San Ángel

Meyer, V. G. 1966. Flower abnormalities. Bot. Rev. 32(2): 165-218.

Mohl, H. von. 1837. Sur la metamorphose des anthéres en carpelles. Ann. Sci. Nat. $2^{\mathrm{c}}$ Sér. 8: 50-75. Seringe, T. 1830. Notice sur une monstruosité du Diplotaxis tenuifolia. Bull. Bot. Génève 1: 5-8.

Sussex, I. M. y N. M. Kerk. 1990. The origin and development of flowers. In: Bennett A. B. y S. O'Neill (eds.). Plant biology. Vol. II. Horticultural biotechnology. Wiley-Liss, Inc. Nueva York. pp. 173182.

Zeevaart, J. A. D. 1978. Phytohormones and flower formation. In: Letham, D. S., P. B. Goodwin y T. J. Higgins (eds.): Phytohormones and related compounds - A comprehensive treatise. Vol. II. Elsevier/North Holland Biomedical Press. Amsterdam. pp. 291-327. 